\title{
THE POSITIVE IMPACT OF AGILE RETROSPECTIVES ON THE COLLABORATION OF DISTRIBUTED DEVELOPMENT TEAMS - A PRACTICAL APPROACH ON THE EXAMPLE OF BOSCH ENGINEERING GMBH
}

\author{
Duehr, Katharina (1); \\ Efremov, Pauline (1); \\ Heimicke, Jonas (1); \\ Teitz, Emilie Maria (2); \\ Ort, Ferdinand (2); \\ Weissenberger-Eibl, Marion (1); \\ Albers, Albert (1) \\ 1: Karlsruhe Institute of Technology (KIT); \\ 2: Bosch Engineering GmbH
}

\begin{abstract}
To counteract competitive pressure, increasing customer requirements and growing product complexity successful distributed collaboration in product development is vital. Companies have to face new challenges, such as efficiency losses in communication. To overcome these challenges agile working practices, such as agile retrospectives, could be beneficial. The objective of this scientific work is to evaluate the benefit of agile working practices on the example of agile retrospectives, for the improvement of collaboration in distributed development teams. Based on literature analysis, qualitative and quantitative expert interviews following the DRM by Blessing and Chakrabarti, this scientific work shows that agile working practices have a high potential to improve distributed collaboration. To address this potential, several virtual agile retrospectives are developed and conducted within a distributed team at Bosch Engineering GmbH. The evaluation of this approach results in a high potential of agile retrospectives indicating an improvement tendency. Especially iteratively implemented virtual agile retrospectives have a positive impact on successful distributed collaboration.
\end{abstract}

Keywords: Collaborative design, New product development, Teamwork, agile working practices, distributed collaboration

\section{Contact:}

Duehr, Katharina

Karlsruhe Institute of Technology (KIT)

IPEK Institute of Product Engineering

Germany

katharina.duehr@kit.edu 


\section{INTRODUCTION}

The product development context becomes increasingly uncertain and complex. The need to adapt to difficulties, such as growing customer requirements and the increasing need to develop products as interacting systems across different domains, is challenging traditional product development (practices) (Keating et al. 2003). As a promising answer to these challenges, companies increasingly extend their product development activities on a global level with distributed teams working all over the world. The global extension results in advantages, such as bringing together various competencies and resources as well as reducing development times (Gaul 2001). However, new difficulties arise as a result of the large distance across time and space between teams in distributed product development. Delayed communication, a wide range of media and tools, coordination problems, and the lack of personal contact can lead to conflicts and prevent teams from working together successfully (Larsson et al. 2003). With the increasing global extension, companies will have to adapt to this trend and improve their expertise and performance regarding distributed collaboration to make distributed product development successful. At this moment however it is not clear how companies can address potential improvements to promote successful collaboration in globally distributed development teams within their individual development context. Agile working practices, which are increasingly applied in product development, can lead to higher flexibility, better responsiveness to change and uncertainty, and improve communication in teams (Schmidt and Paetzold 2016; Atzberger et al. 2020). Especially agile retrospectives, which are regular meetings to inspect and adapt the collaboration and use of methods in the team, can lead to a continuous improvement of collaboration. Thus, agile working practices and agile retrospectives, in particular, have to be examined as a potential solution to improve the collaboration of globally distributed development teams.

\section{STATE OF RESEARCH}

\subsection{Distributed product development}

The idea of the distribution of tasks in organisations dates back many years and is first introduced by Taylor and Roesler (1913). Ehrlenspiel (1995) extends this idea in the context of product development by stating the importance of distributed work for successfully accomplishing complex tasks. Further, Krause et al. (1996) introduces the importance of communication, coordination, and collaboration as three main characteristics of distributed product development and defines distributed product development as the collaboration on subtasks in product development.

With growing globalisation and internationalisation many companies have to face new challenges such as new competitors entering the market, fast-growing and changing markets, and increasing customer requirements leading to products with growing complexity (Grieb 2008; Kern 2005). To secure their competitiveness many companies choose to operate on a global level (Gaul 2001; Larsson et al. 2003; Grieb 2008). The global extension provides benefits bringing together a diversity of competencies and resources (Larsson et al. 2003). This results in a new emphasis on distributed product development as the work on product development activities over different geographically distributed locations (Gierhardt 2001; Kern 2005).

Distributed product development enables the integration of expertise within the company and from external cooperation partners into the development process (Kern 2005). Thus, interdisciplinary knowhow that is necessary to develop innovative products can be pooled (Gaul 2001). Besides, further opportunities such as the reduction of costs and shorter development times due to considering different time zones, local customer proximity, as well as opening up new technologies, can be used simultaneously (Gaul 2001). The success of distributed product development depends on different factors, such as the ability of team members to build trust and manage conflicts in a team (Hinds and Kiesler 2002; Larsson et al. 2003) The results of a study by Seus et al. (2020) focusing on multilocation project management highlight that communication is key to the success of a distributed collaboration. This is supported by Ostergaard and Summers (2009) who state that communication is "particularly important when a development team is distributed". Albers et al. (2020) conduct a literature-based study that results in the identification of 76 influencing factors that are relevant for the success of distributed product development and assign these factors to the three dimensions of technology, organisation and people.

However, temporal, cultural and spatial differences pose new challenges in collaboration (Gaul 2001). For instance, time differences or the use of technology platforms that do not work reliably can 
complicate communication (Larsson et al. 2003). Besides inefficient communication further negative effects, such as coordination problems, conflicts due to a lack of personal contact and delayed resolution of work issues can arise (Larsson et al. 2003). To achieve successful distributed collaboration, it is, therefore, necessary to address these challenges.

\subsection{Agile product development}

Traditional product development is often characterized by planned procedures, such as the stage-gate process, which aim to contribute to its effectiveness and efficiency (Cooper 1994). Product requirements available at the start and a detailed plan for the whole project are important (Petersen and Wohlin 2010). Thus, deviations from the plan are often seen as failure and uncertainties as a vulnerability (Abrahamsson 2002). To increase performance, iterations are often reduced through the development process (Sommer et al. 2015). Also, the development process can be considered as a problem which is a "deviation between the little known actual state and the desired vague target state, linked with the partially unknown path from the actual to the target state" as defined by Albers et al. (2005).

According to Schmidt et al. (2018), the product development context becomes increasingly complex and is shaped by uncertainties and change. This makes the application and management of iterations vital in the development process (Martinez Leon et al. 2013). The application of agile working practices in development teams can help to counteract these difficulties (Nuhn et al. 2016; Schmidt et al. 2018).

Although originally used in software development, the importance of agile working practices and methodologies is thus increasing in physical product development (Goevert et al. 2019).

By providing increasing flexibility through shorter iterations, agile development can lead to higher responsiveness to uncertainties and changes (Nuhn et al. 2016; Schmidt et al. 2018). According to Atzberger et al. (2020), agile methodologies can also improve communication, transparency and increase the satisfaction of the customers' needs and are therefore beneficial in product development. Agile retrospectives, which are iterative team meetings with the main objective to inspect and adapt the collaboration and methods in a team, can help development teams to continuously learn and improve their collaboration (Derby and Larsen 2012). Therefore, the application of agile retrospectives could be in particular beneficial for a successful collaboration of globally distributed teams in product development.

In global development, an extension or modification of agile working practices before the application is important to support the communication of distributed teams (Hossain et al. 2009). The Agile Systems Design (ASD) approach by Albers et al. (2019) serves as a guideline to adapt agile working practices for product development processes and therefore can help distributed teams in selecting a suitable procedure depending on their individual development situation and the level of uncertainty of the development context. A systematic approach developed by Duehr et al. (2019) enables to determine the individual development context based on the degree of complexity and planning stability and thus can help distributed teams to adapt agile practices according to their situation and need. Thereby, an adaptation of agile retrospectives to the individual development context and situation of a team should be considered in the context of improving collaboration in distributed development teams.

\section{RESEARCH OBJECTIVE AND METHODOLOGY}

The state of research indicates a potential of agile working practices to improve the collaboration of development teams. The application of agile retrospectives indicates a particular potential to improve the collaboration of development teams through iterative inspection and adaptation of the collaboration and the methods used within the team (Derby and Larsen 2012). Furthermore, the increasing importance of collaboration in globally distributed development teams and ensuring its success leads to the core objective of this scientific work: Evaluation of the importance and added value of agile working practices on the example of agile retrospectives for the improvement of distributed collaboration in development teams. For this reason, this scientific work is conducted in cooperation with a distributed development team at Bosch Engineering $\mathrm{GmbH}$, which operates in Vehicle Systems Engineering with engineers collaborating in various projects over different domains, to address the research objective in a real development environment. Based on the research objective, the main hypothesis and three research questions to verify the hypothesis are drawn.

- Hypothesis: The application of agile retrospectives has a positive impact on the collaboration within the cooperating distributed development team at Bosch Engineering $\mathrm{GmbH}$. 
- RQ1: How can the general potential of agile working practices to improve success-relevant factors of distributed collaboration be identified at Bosch Engineering $\mathrm{GmbH}$ ?

- RQ2: Which practical approach can be developed to address agile retrospectives to improve distributed collaboration within the cooperating development team at Bosch Engineering $\mathrm{GmbH}$ ?

- RQ3: How does the developed practical approach contribute to the improvement of distributed collaboration within the cooperating development team at Bosch Engineering $\mathrm{GmbH}$ ?

The approach of Design Research Methodology (DRM) by Blessing and Chakrabarti (2009) is used as the framework for this scientific work and consists of the four steps: Research Clarification, Descriptive Study I, Prescriptive Study and Descriptive Study II. First, in the Research Clarification, a literature analysis is conducted to gain an overview of the state of the art on distributed product development, agile working practices, and particularly distributed collaboration of development teams to clarify the research need and derive the research objective. In the next step, within the Descriptive Study I, a mixed-methods study is conducted to generate empirical data to identify the general potential of agile working practices to improve distributed collaboration. This study consists of a quantitative part to get an overview regarding how agile working practices can influence distributed collaboration followed by a qualitative expert interview to get a deeper understanding of the previously obtained data. In the Prescriptive Study, a synthesis of previous findings is used to create a solution approach. To ensure continuous evaluation, the developed approach is evaluated regarding its functionality, completeness, and consistency. Finally, in the Descriptive Study II, a quantitative study is developed to evaluate the applicability and success of the solution approach. This study is conducted with the cooperating development team at Bosch Engineering $\mathrm{GmbH}$ before and after the implementation of the solution approach to ensure its continuous evaluation and to derive opportunities for improvement.

\section{THE POTENTIAL OF AGILE WORKING PRACTICES TO IMPROVE DISTRIBUTED COLLABORATION}

To identify the impact of agile working practices on distributed collaboration and answer the first research question, a mixed-methods study is conducted company-wide at Bosch Engineering GmbH. This study consists of a quantitative part intending to obtain data regarding the influence of agile working practices on distributed collaboration in general and is followed by a qualitative expert interview with the same participants resulting in qualitative statements for a better understanding of the previously obtained data. This study includes 19 participants who are experienced in agile working practices and further chosen for this study based on the following criteria:

- $\quad$ from 13 different departments

- with different context of working with agile practices, such as working in an agile team as a developer, in team coaching, or on the organization development level,

- $\quad$ with the above-average estimated understanding of agile working practices based on individual know-how and experience in this field.

In the quantitative part of the study, the participants have to assess the influence of agile working practices on distributed collaboration, which is represented by preselected factors that are relevant for its success, based on their knowledge and experience in this field. For this reason, 15 success-relevant influencing factors are chosen from the comprehensive list of success-relevant influencing factors identified through a literature-based study by Albers et al. (2020). The pre-selection of success-relevant influencing factors is further based on the following criteria:

- Factors with high relevance for the success of distributed collaboration, assessed by the study of Albers et al. (2020)

- Factors that are presumed to be beneficial through agile working practices based on the results of a study by Atzberger et al. (2020) evaluating agile development of physical products

The 15 following pre-selected success-relevant influencing factors are divided into several dimensions, as communication or interpersonal work, for better differentiation:

- Communication: reliability and frequency of communication

- Interpersonal work: cross-functional interaction; work culture; relationships between teams/ departments

- Characteristics and skills of the team: understanding of the objective; awareness for quality of work 
- Knowledge generation and management: existing knowledge about reference products/projects; knowledge management

- Team internal organisation structures: team organisation; team structures at other locations

- Leadership: leadership styles

- Process organisation: system-wide use of methods

- Project organisation: complexity; customer integration

Further, the influence of agile working practices on each success-relevant factor of distributed collaboration is assessed by the participants over the following two values:

- The direction of the influence, which describes whether the influence on distributed collaboration is positive or negative. Possible answers: [no influence (x), negative influence (-1), neutral influence (0), positive influence (1), no statement possible (n/a)]

- The intensity of the influence, which describes how strong the influence is on distributed collaboration. Possible answers: [no influence (x), low influence (1), medium influence (2), high influence (3), no statement possible (n/a)]

A combination of both values (direction value multiplied with intensity value) leads to the following total influence values: $\mathbf{- 3}$ (high negative influence),-2 (medium negative influence),-1 (low negative influence), $\mathbf{0}$ (neutral influence/ both negative and positive influence possible), $+\mathbf{1}$ (low positive influence), $+\mathbf{2}$ (medium positive influence), $+\mathbf{3}$ (high positive influence). The answers no influence and no statement possible are eliminated and not further examined in the evaluation of the results.

To better illustrate the distribution of the results, in the context of the descriptive study, a boxplot diagram is used with the assessed influence value on the y-axis and the success-relevant factors on the $\mathrm{x}$-axis.

Figure 1 illustrates the results of the assessed influence values per success-relevant influencing factor. The results are structured in decreasing order by a median from left to right and the numbers in the box stand for the median values per influencing factor. Example: For the first success-relevant factor communication, the median value for the assessed influence values equals +3 . This indicates that at least $50 \%$ of the participants think that the application of agile working practices has a highly positive influence on distributed collaboration and thus could lead to its improvement.

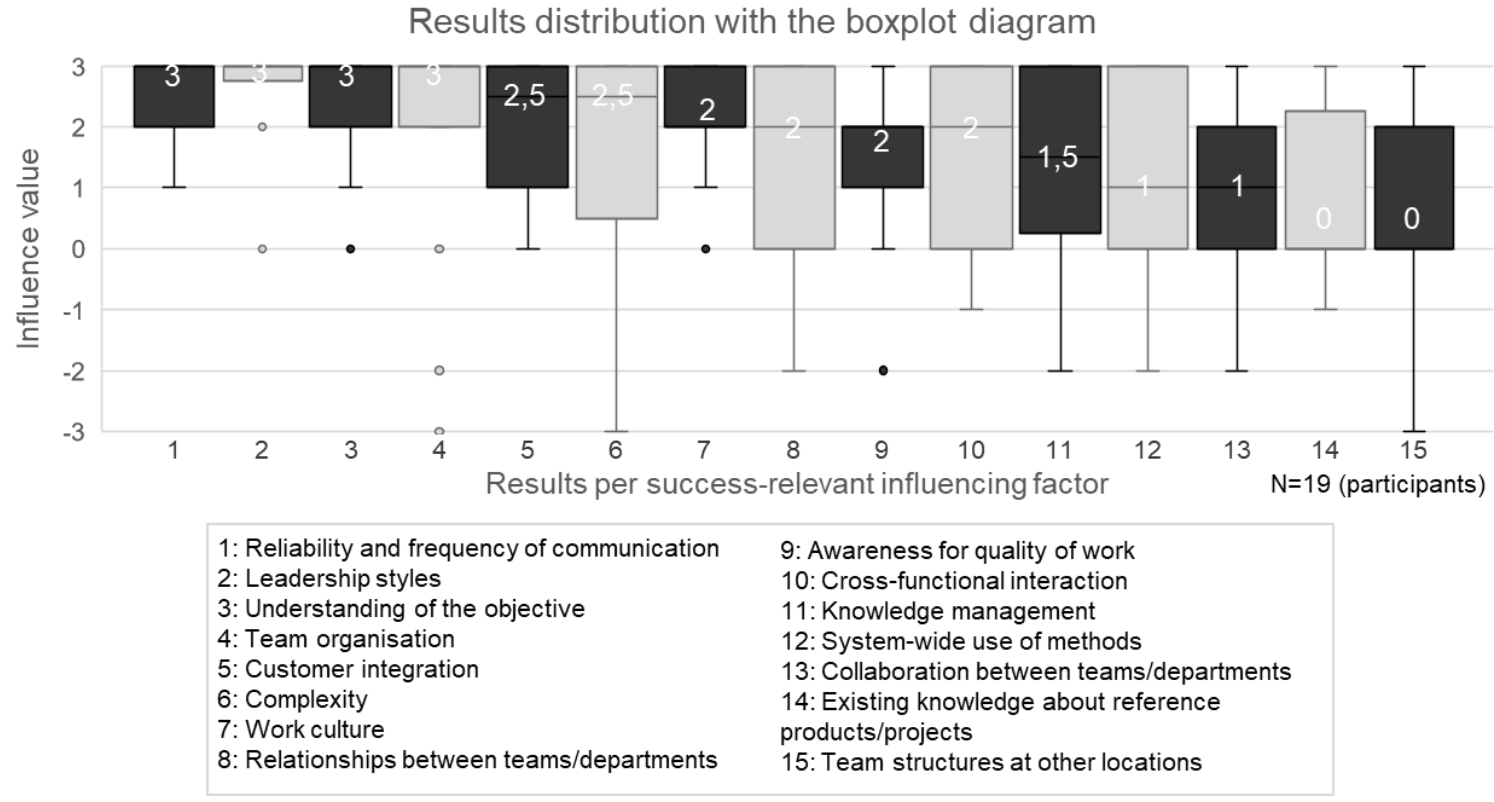

Figure 1. Results distribution (own representation)

In general, all boxes are located above the influence value of 0 , which means that at least $50 \%$ of the participants assessed the influencing value of agile working practices on each factor with a value above 0 , which stands for a positive influence. For 5 out of 15 influencing factors even all results including outliers are above 0 , and median values between 2 and 3 indicating that there is a medium to the high positive influence of agile working practices on the following influencing factors: reliability and frequency of communication, leadership styles, understanding of the objective, customer 
integration and work culture. However, 7 out of 15 influencing factors have lower whiskers that are located below 0 and for the factors complexity and team structure on other locations even at the lowest value of -3 . The lower whisker shows, on one hand, the minimum result value that was assessed by the participants. On the other hand, the lower whisker also shows that $25 \%$ of the results are located between the lower whisker and the box. Thus, the results strongly differ between the participants regarding some influencing factors, such as collaboration between teams/departments, existing knowledge about reference products/projects, team structures at other locations, with long upper and lower whiskers. This indicates that the influence of agile working practices on some influencing factors is either not clear or depends strongly on the individual knowledge and experience of the participants. It should also be noted that results located above 1.5 times the interquartile range are represented as outliers in the boxplot diagram, such as the factors leadership styles or team organisation indicating that for these factors the majority of results were similar with some exceptions. The qualitative statements of the participants resulting from the expert interview help interpret the results. Accordingly, the outliers can be explained by the different individual experience the participants have with agile working practices and the wide range of different possible impacts of the use of agile working practices depending on other general conditions, such as the project type and if the collaborating teams, departments, or the customer also work with agile working practices. In conclusion, the results show that agile working practices generally have a positive influence on distributed collaboration represented through several pre-selected, success-relevant influencing factors which provides an argument to confirm the hypothesis of this scientific work. In addition, some success-relevant factors, such as the reliability and frequency of communication, leadership styles, and work culture can be influenced more positively by agile working practices than others. This is confirmed by the qualitative statements of the participants who highlighted the positive effect of agile working practices facilitating transparency, good communication channels and many exchanges. Besides, three out of nineteen participants stated that especially for the long-term success of distributed collaboration at least some personal (on-site) contact or get-together is highly important both for the success of agile working practices and distributed collaboration.

These results lead to the conclusion that there is a high potential of agile working practices to improve distributed collaboration. This highlights the need to identify an approach to address the potential of agile working practices to improve distributed collaboration and to examine how this approach can be applied and evaluated in a real development environment.

As described before, the state of research regarding agile development indicates a potential of agile retrospectives to improve distributed collaboration due to its focus on reflecting and improving collaboration in development teams. For these reasons, the agile retrospective is chosen as a base for the development of a solution approach as an outcome of the Prescriptive Study to answer the second research question. Furthermore, the solution approach is adapted to the individual situation and needs of the cooperating development team at Bosch Engineering $\mathrm{GmbH}$.

To evaluate if the approach functions as intended a checklist with objectives and measures to conduct an agile retrospective is developed and applied throughout the development of the solution approach. This serves to ensure a continuous evaluation of the approach as a support of the scientific work in the context of the DRM methodology (Blessing and Chakrabarti 2009).

The developed approach consists of two digital agile retrospectives applied twice with a difference of two months in the cooperating development team at Bosch Engineering $\mathrm{GmbH}$. Due to the team members working from different locations the retrospectives are developed in a virtual format using digital tools, such as MS Skype to enhance distributed participation in the retrospective, and MS OneNote to document the resulting improvement measures. Both retrospectives focus on the main objective to improve the collaboration of the team with different focus topics from general collaboration to specific technical exchange formats or team meetings such as virtual coffees. As a result of each retrospective, several improvement measures were derived to be implemented in the team until the next agile retrospective. As an example, a regular technical exchange was implemented to enhance the collaboration on technical topics. In addition, small groups were formed to move different improvement measures regarding topics, such as goals, project infights, and competencies in the team, forward. 


\section{THE POSITIVE IMPACT OF RETROSPECTIVES ON DISTRIBUTED COLLABORATION}

To ensure a continuous evaluation of the developed approach and answer the third research question, a quantitative study is conducted with 8 team members before and after the second retrospective to include the effect of the retrospective as an iterative meeting. The focus of this study in the context of the DRM methodology lies in evaluating the applicability and success of the retrospective to examine how good the agile retrospective can be used by the participants and which added value it can provide regarding its intended objectives. To better evaluate the success of the retrospective regarding different aspects of distributed collaboration which are derived from the success-relevant influencing factors by Albers et al. (2020), the success evaluation is extended with the following questions and possible answers on a 5-point Likert scale:

- How is the currently distributed collaboration in your team? [very bad - very good]

- How is the reliability of current communication in your team? [very low - very high]

- How often does an exchange take place (frequency)? [too rare - too often] It should be noted that the optimal answer is 3: as often, as necessary.

- How is the quality of the current exchange in the team? [very bad - very good]

- How is the trust in the relationship between team members? [not openly/trusting -openly/trusting]

- How is the common understanding of objectives in the team? [different - uniform]

- How is the clarity of objectives within the team? [unclear - clear]

- How is the transparency of information and knowledge in the team? [non-transparent - transparent]

- How often are information and knowledge shared in the team (frequency)? [too rare, rather too rare, as often as necessary, rather too often, too often] It should be noted that the optimal answer is 3: as often, as necessary.

- How is the variety of tools used in the team? [too low - too high] It should be noted that the optimal answer is 3 as high, as necessary.

- How is the reliability of the tools used in the team? [worse than expected - better than expected]

The results regarding different aspects of distributed collaboration are shown in Figure 2, which represents the distribution of results, including a representation of the mean values in numbers and the spread of the data over standard deviations shown as whiskers, before and after the retrospective. The different answers (from 1 to five) are shown on the y-axis and the different aspects of collaboration can be found on the $\mathrm{x}$-axis:
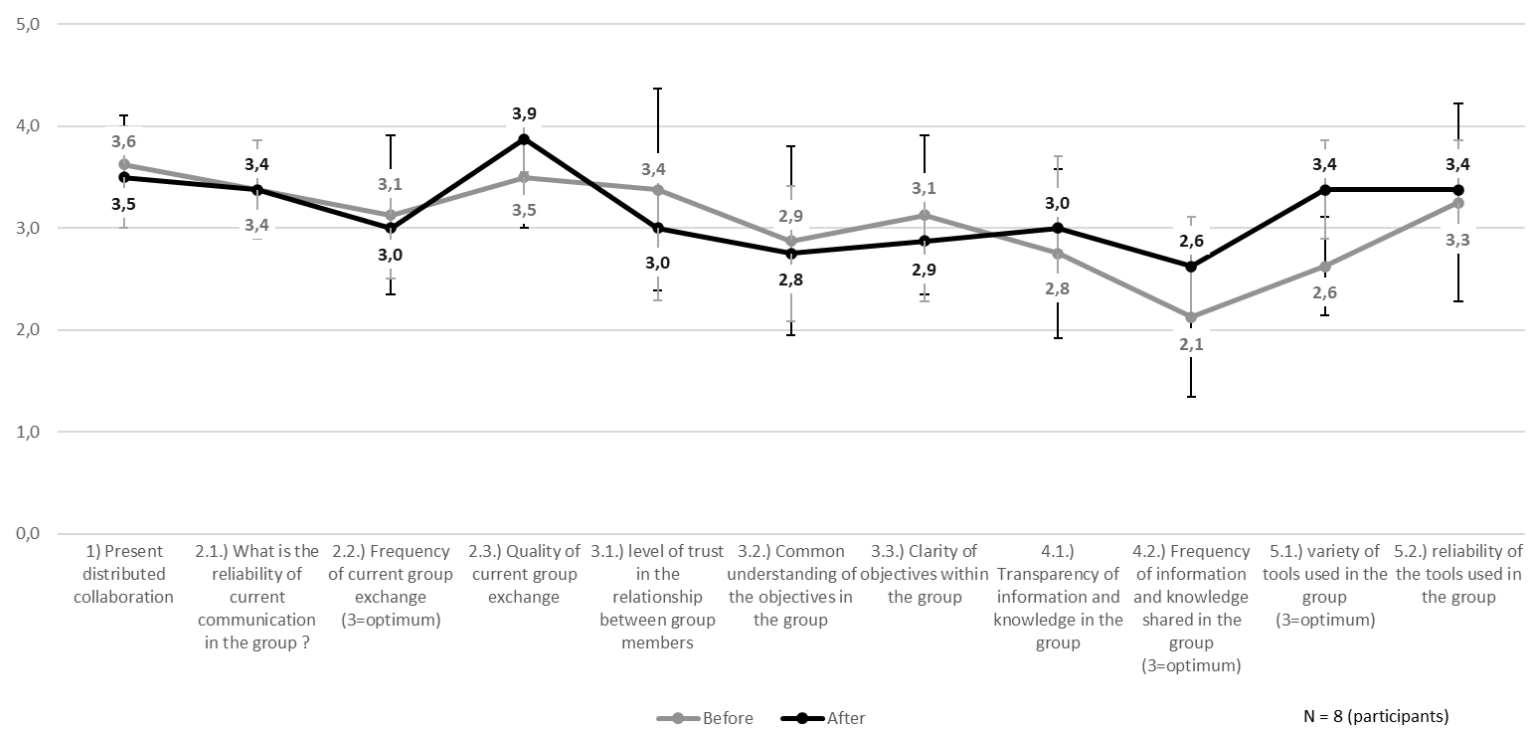

Figure 2. Comparison of distributed collaboration before and after the application of the developed approach (own representation)

These results show that the currently distributed collaboration was rated similar after the retrospective compared to before. Other aspects such as the reliability of current communication, the common understanding, and clarity of the objectives or the reliability of the tools used in the team, have not changed after the application of the retrospectives. Positive effects could be achieved regarding the 
quality of the team exchange, which increased from a mean value of 3,5 to 3,9, the frequency of information and knowledge shared which increased from a mean value of 2,1, which stand for rather to rare, to 2,6, which is closer to the optimum of 3 which stands for as often as necessary. Besides, only one aspect level of trust in the relationship between team members was assessed as being less good after the retrospective compared to before with a mean value shift from 3,4 to 3,0. Regarding the spread of the data, the standard deviations are in general higher before compared to after the application of agile retrospectives, indicating that the participants had fewer different answers regarding the aspects of their collaboration. For these reasons, these results indicate an improvement tendency regarding some aspects of distributed collaboration after applying the retrospectives compared to before.

The results focusing on the general success evaluation show that the majority (88\%) of participants think that the retrospective has led to an improvement in the distributed collaboration of their team, which supports the perceived improvement tendency regarding the evaluation of different aspects of distributed collaboration. The majority (63\%) of participants perceived the satisfaction with the implemented measures, which resulted from the retrospectives, as good. In conclusion, the developed approach and thereby agile retrospectives, in general, have a positive impact on distributed collaboration. The results of the study regarding the applicability evaluation indicate that all participants agree that the feasibility of the developed approach is good. Also, more than $63 \%$ of the participants state that the retrospective addresses the reflection and improvement of distributed collaboration better than expected, which is a good result since they are key aspects of a retrospective and therefore should be addressed correctly for its success. In addition, the retrospective has influenced the individual reflection and improvement of the distributed collaboration of the participating team in an overall positive way according to the majority (63\%) of the participants.

\section{DISCUSSION}

The first study regarding the influence of agile working practices on the selected success-relevant influencing factors of distributed collaboration resulted in most of the participants assessing a positive influence for all influencing factors. This result indicates a high potential of agile working practices to improve distributed collaboration in general. However, the results highlight differences regarding different influencing factors of distributed collaboration, such as communication or knowledge management. This leads to the assumption that not all aspects of distributed collaboration can be influenced equally positively by agile working practices. Besides, some outliers were found in the results which can be explained by the different individual experience the participants have with agile working practices and the wide range of different possible impacts of the use of agile working practices depending on other general conditions, such as the project type and if the collaborating teams, departments or the customer also work with agile working practices. Furthermore, some influencing factors can be influenced more positively by agile working practices, such as the reliability and frequency of communication, understanding of the objective in the team, or customer integration.

The evaluation of the developed approach to address agile working practices as a result of the identified potential of agile working practices to improve distributed collaboration shows that the conducted retrospective has good applicability addressing the necessary aspects of collaboration. Regarding the success evaluation, the before and after comparison of specific aspects in distributed collaboration in the team resulted in a rather similar assessment of distributed collaboration with few improved aspects, such as a better quality of team exchanges and a better variety of tools used within the team. The small improvement tendency could be explained by various reasons, such as the team members working on different projects and focusing more on the project work than implementing the improvements developed in the retrospectives. Further, most of the team members were new to agile retrospectives and thus needed time to get accustomed to the procedure and sharing their ideas. In the considered period of two months, only two retrospectives could be conducted making it difficult to implement more extensive improvement measures which require a longer period to be implemented or require major changes to work.

The different results support the previous assumption that the influence of agile working practices on distributed collaboration differs between the success-relevant aspects. Despite the rather small improvement tendency regarding aspects of distributed collaboration and the short period of the study, the general success of agile retrospectives to improve distributed collaboration was assessed positively and the team members were satisfied with the implemented improvement measures. Thus, these 
positive results indicate that agile retrospectives have a high improvement potential regarding distributed collaboration with an increasing improvement tendency over time.

\section{CONCLUSION \& OUTLOOK}

This scientific work indicates that agile working practices such as retrospectives have a high potential to improve distributed collaboration, which is identified through a mixed-methods study including a quantitative part and a qualitative expert interview with 19 participants at Bosch Engineering. To address this potential a practical solution approach was developed consisting of the application of two agile retrospectives in virtual format within a distributed development team operating in Vehicle Systems Engineering at Bosch Engineering GmbH. The developed and applied agile retrospectives are evaluated regarding applicability and success over a quantitative study conducted before and after the application of the agile retrospectives. The results of the study indicate an improvement tendency due to agile retrospectives and thus a positive impact of agile retrospectives regarding distributed collaboration. However, the results also indicate that it needs more time than the period considered in this scientific work to achieve major improvements in distributed collaboration, which introduces the need for further research in this field to evaluate the long-term influence of agile retrospectives on distributed collaboration. In addition, this approach is only focusing on agile retrospectives as an example for agile working practices due to its focus on general collaboration in development teams. For this reason, further research evaluating the impact of other agile elements or procedures such as Scrum to improve distributed collaboration is necessary. In this context, it is important to proceed in a structured manner identifying and analysing the specific individual situation regarding distributed collaboration first and applying the right method next. Thus, an approach supporting this procedure that focuses on improvement potentials in distributed collaboration could be beneficial as confirmed and examined by Duehr et al. (2020).

Distributed collaboration represents the fundamental collaboration model in ASE - Advanced Systems Engineering for the development of intelligent and connected systems. Through the systematic implementation of agile methods, it is ultimately possible to design the collaboration of all participants efficiently and effectively for the individual development situation.

\section{REFERENCES}

Abrahamsson, Pekka (2002): Agile software development methods. Review and analysis. Espoo: VTT (VTT publications, 478). Available online at https://arxiv.org/abs/1709.08439.

Albers, A.; Burkhardt, N.; Meboldt, M.; Saak, M. (2005): SPALTEN Problem Solving Methodology in the Product Development. In : Engineering Design and the Global Economy: 15th International Conference on Engineering Design - ICED 2005, Melbourne, 15. - 18. August 2005. Hrsg.: A. Samuel: The Design Society, Melbourne, p. 90671.

Albers, Albert; Heimicke, Jonas; Spadinger, Markus; Reiss, Nicolas; Breitschuh, Jan; Richter, Thilo et al. (2019): A systematic approach to situation-adequate mechatronic system development by ASD - Agile Systems Design.

Albers, Albert; Weissenberger-Eibl, Marion A.; Duehr, Katharina; Zech, Katharina; Seus, Fanny (2020): Literature-based identification of success-relevant influencing factors of distributed product development. In 30th CIRP Design 2020 (CIRP Design 2020).

Atzberger, Alexander; Nicklas, Simon J.; Schrof, Julian; Weiss, Stefan; Paetzold, Kristin (2020): Agile Entwicklung physischer Produkte. Eine Studie zum aktuellen Stand in der industriellen Praxis. Neubiberg: Universitätsbibliothek der Universität der Bundeswehr München.

Beck, K.; Beedle, M.; van Bennekum, A.; Cockburn, A.; Cunningham, W.; Fowler, M. et al. (2001): Agile Manifesto. Available online at https://agilemanifesto.org/, checked on 10/13/2020.

Blessing, Lucienne T.M.; Chakrabarti, Amaresh (2009): DRM, a Design Research Methodology. London: Springer London. Available online at http://site.ebrary.com/lib/alltitles/docDetail.action?docID=10310350.

Cooper, R. (1994): Perspective third-generation new product processes. In J Product Innovation Man 11 (1), pp. 3-14. DOI: 10.1016/0737-6782(94)90115-5.

Derby, Esther; Larsen, Diana (2012): Agile retrospectives. Making good teams great. Bookversion: P7.0. Dallas, Texas, Raleigh, North Carolina: The Pragmatic Bookshelf (The pragmatic programmers).

Duehr, K.; Hirsch, M.; Albers, A.; Bursac, N. (2020): A methodology to identify and address improvement potentials in communication processes of distributed product development - an initial approach. In Proc. Des. Soc.: Des. Conf. 1, pp. 541-550. DOI: 10.1017/dsd.2020.35. 
Duehr, Katharina; Heimicke, Jonas; Breitschuh, Jan; Spadinger, Markus; Kopp, David; Haertenstein, Lars; Albers, Albert (2019): Understanding Distributed Product Engineering: Dealing with Complexity for Situation- and Demand-Oriented Process Design. In Procedia CIRP 84, pp. 136-142. DOI: 10.1016/j.procir.2019.04.200.

Ehrlenspiel, Klaus (1995): Integrierte Produktentwicklung. Methoden für Prozeßorganisation, Produkterstellung und Konstruktion. München: Hanser.

Gaul, Hans-Dieter (2001): Verteilte Produktentwicklung. Perspektiven und Modell zur Optimierung. Zugl.: München, Techn. Univ., Diss., 2001. 1. Aufl. München: Hut (Produktentwicklung).

Gierhardt, Heiko (2001): Global verteilte Produktentwicklungsprojekte. Dissertation. Technische Universität München, München.

Goevert, Kristin; Heimicke, Jonas; Lindemann, Udo; Albers, Albert (2019): Interview Study on the Agile Development of Mechatronic Systems. In Proc. Int. Conf. Eng. Des. 1 (1), pp. 2287-2296. DOI: 10.1017/dsi.2019.235.

Grieb, Jöran Christopher (2008): Auswahl von Werkzeugen und Methoden für verteilte Produktentwicklungsprozesse. Zugl.: München, Techn. Univ., Diss., 2007. 1. Aufl. München: Dr. Hut (Produktentwicklung).

Hinds, Pamela; Kiesler, Sara (2002): Distributed work. Cambridge, Mass: MIT Press. Available online at http://search.ebscohost.com/login.aspx?direct=true\&scope=site \&db=nlebk\&db=nlabk\&AN=70964.

Hossain, Emam; Babar, Muhammad Ali; Paik, Hye-young (2009): Using Scrum in Global Software Development: A Systematic Literature Review. In 4th IEEE International Conference on Global Software Engineering, pp. 175-184. DOI: 10.1109/ICGSE.2009.25.

Keating, Charles; Rogers, Ralph; Unal, Resit; Dryer, David; Sousa-Poza, Andres; Safford, Robert et al. (2003): System of Systems Engineering. In Engineering Management Journal 15 (3), pp. 36-45. DOI: 10.1080/10429247.2003.11415214.

Kern, Eva-Maria (2005): Verteilte Produktentwicklung - Rahmenkonzept und Vorgehensweise zur organisatorischen Gestaltung. Zugl.: Hamburg, Techn. Univ., Habil.-Schr., 2005. Berlin: GITO Verl. für industrielle Informationstechnik und Organisation.

Krause, F. L.; Jansen, H.; Kiesewetter, T. (1996): Verteilte, kooperative Produktentwicklung durch Integration heterogener CAD-Systeme in eine multimediale Breitbandkommunikationsumgebung, Distributed, cooperative product development, ZWF Zeitschrift für wirtschaftlichen Fabrikbetrieb. In ZWF Zeitschrift für wirtschaftlichen Fabrikbetrieb 91 (4), pp. 147-151. Available online at https://www.tib.eu/de/suchen/id/tema\%3ATEMAM96050492683.

Larsson, Andreas; Törlind, Peter; Karlsson, Lennart; Mabogunje, Ade; Leifer, Larry; Larsson, Tobias; Elfstrm, Bengt-Olof (2003): Distributed team innovation - a framework for distributed product development, pp. 321-322.

Martinez Leon, Hilda C.; Farris, Jennifer A.; Letens, Geert (2013): Improving Product Development Performance Through Iteration Front-Loading. In IEEE Trans. Eng. Manage. 60 (3), pp. 552-565. DOI: 10.1109/TEM.2012.2228205.

Nuhn, H. F.; Martini, J.-P; Kostron, A. (2016): Hybride Strukturen in der Automobilindustrie - Studie zu Agilen Praktiken in Forschungs- und Entwicklungsprozessen. In Engstler, M., Fazal-Baqaie, M., Hanser, E., Linssen, O., Mikusz, M. \& Volland, A. (Hrsg.), Bonn: Gesellschaft für Informatik e.V., pp. 29-36.

Ostergaard, Karen J.; Summers, Joshua D. (2009): Development of a systematic classification and taxonomy of collaborative design activities. In Journal of Engineering Design 20 (1), pp. 57-81. DOI: $10.1080 / 09544820701499654$.

Petersen, Kai; Wohlin, Claes (2010): The effect of moving from a plan-driven to an incremental software development approach with agile practices. In Empir Software Eng 15 (6), pp. 654-693. DOI: 10.1007/s10664-010-9136-6.

Schmidt, Tobias; Weiss, Stefan; Paetzold, Kristin (2018): VDI-Statusreport Agile Entwicklung physischer Produkte.

Schmidt, Tobias Sebastian; Paetzold, Kristin (2016): Agilität als Alternative zu traditionellen Standards in der Entwicklung physischer Produkte: Chancen und Herausforderungen. In Dieter Krause, Kristin Paetzold, Sandro Wartzack (Eds.): Design for X - Beiträge zum 27. DfX-Symposium Oktober 2016. Hamburg: TuTech Verlag TuTech Innovation GmbH, pp. 255-267.

Seus, Fanny; Weissenberger Eibl, Marion A.; Zern Breuer, Rubina (2020): Considering representational gaps how subsidiaries' relationship affects multi-location project management. In IJPOM 12 (4), p. 321. DOI: 10.1504/IJPOM.2020.10033026.

Sommer, Anita Friis; Hedegaard, Christian; Dukovska-Popovska, Iskra; Steger-Jensen, Kenn (2015): Improved Product Development Performance through Agile/Stage-Gate Hybrids: The Next-Generation Stage-Gate Process? In Research-Technology Management 58 (1), pp. 34-45. DOI: 10.5437/08956308X5801236.

Taylor, Frederick Winslow; Roesler, Rudolf (1913): Die Grundsätze wissenschaftlicher Betriebsführung. [Reprint der Ausg. 2013] München, Berlin 1913. Paderborn: Salzwasser-Verl. 